\title{
Sensitivity of Nitrogen K-Edge X-ray Absorption to Halide Substitution and Thermal Fluctuations in Methylammonium Lead- Halide Perovskites
}

Cody M. Sterling, Chinnathambi Kamal, Gabriel J. Man, Pabitra K. Nayak, Konstantin A. Simonov, Sebastian Svanström, Alberto García-Fernández, Thomas Huthwelker, Ute B. Cappel, Sergei M. Butorin, Håkan Rensmo, and Michael Odelius*

Cite This: J. Phys. Chem. C 2021, 125, 8360-8368

Read Online

ABSTRACT: The performance of hybrid perovskite materials in solar cells crucially depends on their electronic properties, and it is important to investigate contributions to the total electronic structure from specific components in the material. In a combined theoretical and experimental study of $\mathrm{CH}_{3} \mathrm{NH}_{3} \mathrm{PbI}_{3}$ - methylammonium lead triiodide (MAPI) - and its bromide cousin $\mathrm{CH}_{3} \mathrm{NH}_{3} \mathrm{PbBr}_{3}$ (MAPB), we analyze nitrogen K-edge ( $\mathrm{N}$ 1s-to$2 \mathrm{p}^{*}$ ) X-ray absorption (XA) spectra measured in MAPI and MAPB single crystals. This permits comparison of spectral features to the local character of unoccupied molecular orbitals on the $\mathrm{CH}_{3} \mathrm{NH}_{3}{ }^{+}\left(\mathrm{MA}^{+}\right)$counterions and allows us to investigate how thermal fluctuations, hydrogen bonding, and halide-ion substitu-

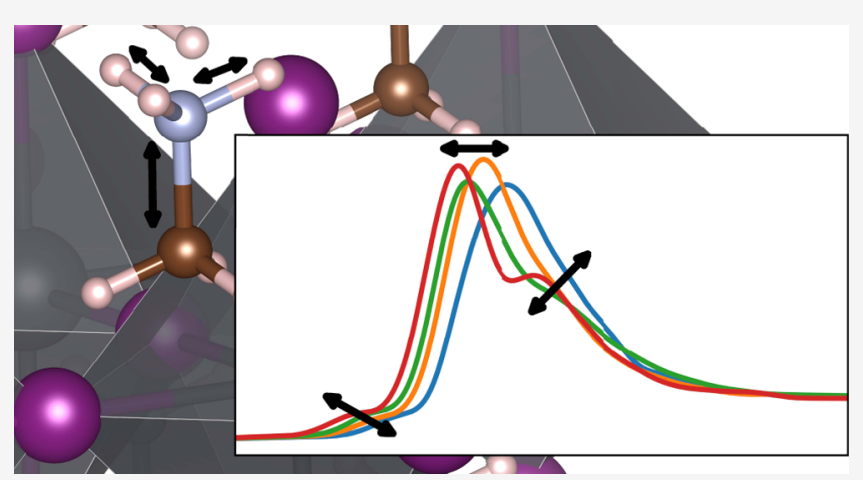
tion influence the XA spectra as a measure of the local electronic structure. In agreement with the experiment, the simulated spectra for MAPI and MAPB show close similarity, except that the MAPB spectral features are blue-shifted by $+0.31 \mathrm{eV}$. The shift is shown to arise from the intrinsic difference in the electronic structure of the two halide atoms rather than from structural differences between the materials. In addition, from the spectral sampling analysis of molecular dynamics simulations, clear correlations between geometric descriptors $(\mathrm{N}-\mathrm{C}, \mathrm{N}-\mathrm{H}$, and $\mathrm{H} \cdots \mathrm{I} / \mathrm{Br}$ distances $)$ and spectral features are identified and used to explain the spectral shapes.

\section{INTRODUCTION}

Halide perovskite materials, especially hybrid organicinorganic perovskites, have in recent years been the focus of intense research as solar cells. Perovskites have the general formula $\mathrm{ABX}_{3}$, where the counterion $\mathrm{A}^{+}$lies within the lattice of $\mathrm{BX}_{3}^{-}$. Hybrid perovskites contain an organic counterion $\left(\mathrm{A}^{+}\right)$in cuboctahedral cages in the inorganic metal-halide lattice; the prototypical example is $\mathrm{CH}_{3} \mathrm{NH}_{3} \mathrm{PbI}_{3}$, methylammonium lead triiodide (MAPI), which has been extensively studied. ${ }^{1-3}$ Closely related systems with different counterions or halides are also studied. ${ }^{4-6}$ This class of materials has been shown to have excellent optoelectronic properties ${ }^{7}$ and reach a power conversion efficiency of over $25 \%,{ }^{8}$ but many key questions remain open. For example, it is unclear to what extent the $\mathrm{A}^{+}$counterion is important in the optoelectronic response and subsequent processes, or specifically, how much the lattice (construction and physical dynamics) of the material can change the electronic properties. ${ }^{9}$

In particular, previous work by Lindblad et al. suggests that an increase of electron-binding energy in the valence band of $\mathrm{CH}_{3} \mathrm{NH}_{3} \mathrm{PbBr}_{3}$ (MAPB) compared to MAPI is due to electronic properties of the two halides as opposed to geometric factors. ${ }^{4}$ This suggests that the choice of halide is an important factor when designing a solar cell from this class of materials. An investigation by Wang et al. shows how the band gap of mixed-halide MAPB/I and MAPB $/ \mathrm{CH}_{3} \mathrm{NH}_{3} \mathrm{PbCl}_{3}$ can be tuned by adjusting the ratio of the two halides. ${ }^{10}$ These studies point toward important changes in the electronic structure when varying the halides in perovskites.

Others have investigated the geometric effects on the systems. Svane et al. investigated formate-based hybrid zinc perovskites with differing numbers of hydrogen bond donation sites in the formate counterion, demonstrating a positive correlation between the number of donating sites and the phase transition temperature. ${ }^{11}$ Herz and co-workers have

Received: March 6, 2021

Revised: $\quad$ March 24, 2021

Published: April 9, 2021 
investigated how lattice dynamics influences the electronic properties of pure metal-halide perovskites, indicating how lighter metals in the lattice lead the system to have lower exciton binding energies. ${ }^{9}$

$\mathrm{X}$-ray absorption (XA) spectroscopy is a powerful probe of unoccupied orbitals in a system, and tuning the excitation energy in XA spectroscopy (XAS) to different core levels allows for an element specific analysis of complex materials. At the K-edge, XA spectra can often be readily assigned in a one electron picture, if the influence of core-hole relaxation is taken into account. Furthermore, since the $1 \mathrm{~s}$ core levels are not influenced by spin-orbit coupling, relativistic effects have a small effect on the spectral shape for systems involving low- $Z$ elements. The spectral features are associated with core-excited states, which in electronic structure calculations can be assigned to transitions from a $1 \mathrm{~s}$ core level into unoccupied molecular orbitals with dipole transition probability related to the local $\mathrm{p}$ character of the orbitals. Because of the spatial extent of the unoccupied orbitals, the core-excited states are strongly influenced by coordination and chemical bonding. XA spectroscopy is a very sensitive chemical probe. In analogy to our measurements on the $\mathrm{MA}^{+}$ion, previous work on ammonia and ammonium in aqueous solution indicates an effect of hydrogen bonding on the pre-edge features and overall spectral broadening to explain differences in the ammonia and ammonium N 1s XA spectra in water. ${ }^{12}$ Analogous studies of a series of ethylammonium with $\mathrm{Et}_{y} \mathrm{NH}_{4-y}{ }^{+}(y=0 . . .4$; Et $=$ $\mathrm{C}_{2} \mathrm{H}_{5}$ ) in solution is of direct relevance for comparison to our present data. ${ }^{13}$ Additionally, recently, Kot et al. showed that nitrogen does cause a noticeable change in the electronic structure of the MAPI system using X-ray photoelectron spectroscopy (XPS) and XAS on the N 1s edge. ${ }^{3}$

Complementary to the previous work of Lindblad et al. which primarily investigated the effects of halide substitution on occupied valence states ${ }^{4}$ and the work of Drisdell et al. on geometric effects in MAPI and MAPB on the $\mathrm{Pb} \mathrm{L}_{3}$ absorption edge, ${ }^{14}$ in this paper, we examine unoccupied states with experimental $\mathrm{N}$ 1s XA spectra for MAPB and MAPI single crystals. The experimental spectra are assigned by comparison to computed spectra sampled from $a b$ initio molecular dynamics (AIMD) trajectories. The sampled theoretical spectra are analyzed for correlations with several geometric descriptions by sorting individual spectra along specific geometric coordinates, namely, bond lengths of covalent and hydrogen bonds, and the spectral response and assignments are presented and discussed. A main motivation for this work is to obtain a more fundamental understanding of these changes by studying $\mathrm{N}$ 1s XA spectra from a geometric point of view, that is, how spectral changes can be explained in terms of geometric coordinates such as interatomic distances and asymmetries. We also aim to determine and understand the spectral differences between MAPI and MAPB.

\section{METHODS}

Crystal Growth. Single-crystal cuboids of MAPB and MAPI with edge lengths of approximately $0.5 \mathrm{~cm}$ were obtained by a slow evaporation method. For MAPB, a 1.0 molar precursor solution was prepared by dissolving lead bromide and methylammonium bromide $(1: 1)$ in $N, N$ dimethylformamide. For MAPI, a 1.0 molar precursor solution was prepared by dissolving lead iodide and methylammonium iodide $(1: 1)$ in $\gamma$-butyrolactone. The resulting solutions were filtered with a $0.45 \mu \mathrm{m}$ filter. Small single crystals began to grow at the bottom of the glass vials containing the solutions after slow evaporation of the solvent at $80{ }^{\circ} \mathrm{C}$ for MAPB and at $100{ }^{\circ} \mathrm{C}$ for MAPI. Seed crystals were collected and carefully placed in new glass vials containing filtered solution and were then allowed to grow into larger perovskite crystals. This step was repeated until crystals of the desired size $(0.5 \mathrm{~cm})$ were obtained; in this case, it took three times.

Experimental XA Spectra. For the total electron yield (TEY) XA measurements, the crystals were epoxy-bonded to the sample plates and blade-cleaved in ultrahigh vacuum (UHV). The cleanliness of the cleaved crystal surfaces was checked using XPS with an excitation energy of $535 \mathrm{eV}$, making the measurements highly surface sensitive with an estimated probing depth between $2 \mathrm{~nm}$ for the $\mathrm{N} 1 \mathrm{~s}$ core level and $5 \mathrm{~nm}$ for the valence band and XAS. ${ }^{15,16}$ The C $1 \mathrm{~s}$ spectra showed no feature at energies expected for adventitious carbon from organic contamination but do show a feature from the methylammonium cation. Furthermore, the $\mathrm{N} \mathrm{1s,} \mathrm{Pb} 4 \mathrm{f}, \mathrm{Br} 3 \mathrm{~d}$, and I 4d spectra show only features expected from the perovskite; see Figure S1. The XAS measurements were performed on fresh spots, and the duration of the measurement was less than $30 \mathrm{~min}$. No effect of the beam is expected in the spectra as we previously measured similar samples at the LowDosePES endstation for $7 \mathrm{~h}$ without any measurable change. ${ }^{17}$ TEY nitrogen 1s-to-2p* (N K-edge) XA measurements were performed at the synchrotron BESSY, at the soft $\mathrm{X}$-ray beamline PM4, and at the LowDosePES endstation. The XAS measurements were performed with a photon bandwidth/ resolution $125 \mathrm{meV}$ (at the $\mathrm{N}$ K-edge) at room temperature in a UHV chamber with a base pressure of $1 \times 10^{-9}$ mbar. Photon energy calibration was performed by measuring the kinetic energies of $\mathrm{Au} 4 \mathrm{f}$ photoelectrons excited by first- and second-order light and taking the difference, both below and above the absorption edge. Finally, a straight line background was subtracted.

Molecular Dynamics. AIMD simulations for supercells of MAPI and MAPB in periodic boundary conditions were performed in the $\mathrm{CP} 2 \mathrm{~K}$ code ${ }^{18}$ under the same conditions with different starting supercell structures, as explained below. NPT simulations at $300 \mathrm{~K}$ and 0 atm were performed for 50 ps with a $0.5 \mathrm{fs}$ timestep using orthorhombic simulation cells with fixed cell side ratios. Temperature was maintained using a four-chain Nosé-Hoover thermostat ${ }^{19}$ with a coupling time of $20 \mathrm{fs}$. The barostat time constant was also $20 \mathrm{fs}$ and used a temperature tolerance of $100 \mathrm{~K}$.

Forces for the dynamics were obtained using density functional theory (DFT)-based electronic structure calculations, with the $\mathrm{PBE}^{20}$ exchange-correlation functional and Grimme's D3 van der Waals correction. ${ }^{21,22}$ The sampling of the electronic wave function was restricted to the $\Gamma$ Brillouin zone point. The Gaussian and plane wave (GPW) method ${ }^{23}$ was used with a multigrid consisting of five grids and a cutoff of 600 Rydberg in combination with Goedecker-Teter-Hutter (GTH) pseudopotentials. ${ }^{24,25}$ Corresponding basis sets used were TZVP-MOLOPT-GTH for $\mathrm{C}, \mathrm{N}$, and $\mathrm{H}$ and DZVPMOLOPT-SR-GTH for $\mathrm{Pb}$ and $\mathrm{I} / \mathrm{Br}^{24,26}$

Initial cell parameters were taken from Poglitsch and Weber's study on various perovskite compositions and phases. $^{27}$ For MAPI, we generated a $2 \times 2 \times 2$ supercell from the unit cell of the tetragonal $\beta$ phase $(a=b=8.855 \AA$; $c$ $=12.659 \AA)$. For MAPB, a supercell of comparable size $(3 \times 3$ $\times 4)$ was generated from the unit cell of the cubic $\alpha$ phase $(a=$ $b=c=5.901 \AA)$. The $\mathrm{MA}^{+}$ions were centered in the cavities of 
the inorganic $\mathrm{PbI}_{3}$ lattice, with the $\mathrm{N}-\mathrm{C}$ bond aligned along the $z$ axis. The starting geometries for MAPI and MAPB are shown in Figure 1.

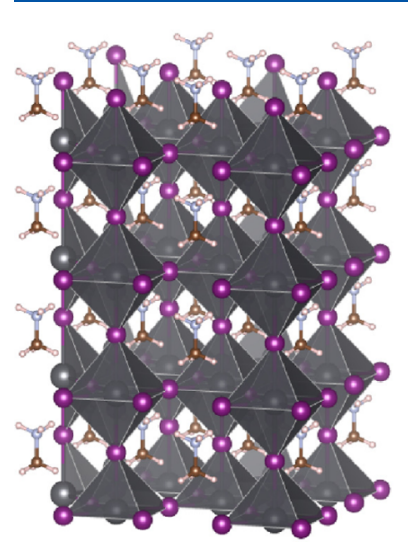

(a)

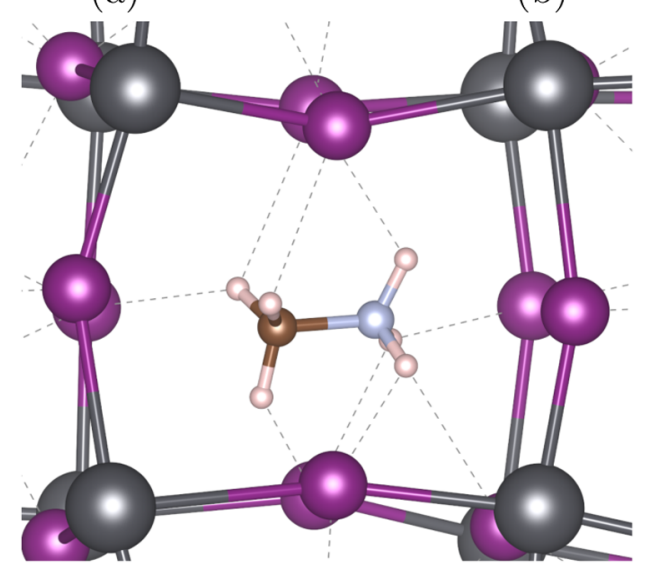

(c)

Figure 1. Starting supercell geometries for (a) $2 \times 2 \times 2$ tetragonal $\beta$ MAPI and (b) $3 \times 3 \times 4$ cubic $\alpha$-MAPB, both with $\mathrm{MA}^{+}$counterions aligned along the $z$ axis. (c) shows a local orientation of the $\mathrm{MA}^{+}$ion in MAPI during the course of the molecular dynamics simulation, with distances between the hydrogens and iodines shown with dashed lines. In $(\mathrm{a}-\mathrm{c})$, carbons are dark brown, nitrogens are blue, hydrogen is white, and lead is gray. Iodine is purple in $(a, c)$, and bromine is light brown in (b).

Computational XA Spectra. Snapshots of the cell geometries were sampled for five configurations at regular intervals during the molecular dynamics simulations. $\mathrm{N}$ 1s XA spectra, obtained within the half core-hole transition potential (TP_HH) approximation, were calculated for each nitrogen present in the supercell, yielding an overall sampling of 160 spectra for MAPI and 180 for MAPB. Inner-shell spectroscopies are enabled by all-electron calculations in the framework of the Gaussian augmented plane wave method, ${ }^{28}$ and we used the same DFT functional and settings as in the GPW-based AIMD simulation, except that a full potential description and all-electron basis sets were used for $\mathrm{C}, \mathrm{N}$, and $\mathrm{H}$ (6-311+ + G2d2p), whereas the TZVP-MOLOPT-SR-GTH pseudopotential basis sets and pseudopotential description were retained for $\mathrm{Pb}$ and $\mathrm{I} / \mathrm{Br}$. Gaussian convolution on the discrete transitions was performed using the normalized Gaussian curve with $\sigma=0.2 \mathrm{eV}$ (full width at half-maximum as
$2 \sqrt{2 \ln 2 \sigma} \approx 0.471 \mathrm{eV})$. Due to limitations of the $\mathrm{XA}$ spectrum simulations, an ad-hoc shift of $-2.86 \mathrm{eV}$ was added to the calculated MAPI and MAPB spectra for direct comparison to the experiment.

\section{RESULTS AND DISCUSSION}

Figure 2 shows the comparison between the experimental nitrogen K-edge XA spectra of MAPB and MAPI and

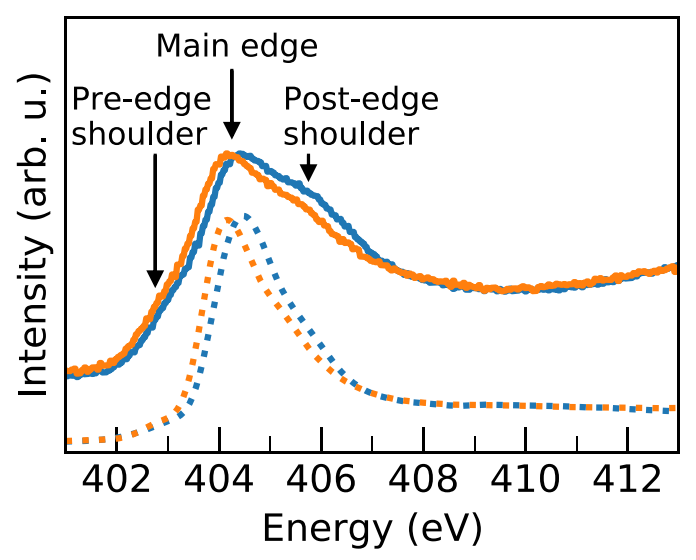

Figure 2. Comparison of experimental N K-edge XA spectra with the TEY for MAPB (solid blue) and MAPI (solid orange) with the corresponding calculated spectra for MAPB (blue dash) and MAPI (orange dash) shifted by $-2.86 \mathrm{eV}$. The arrows indicate the pre-edge shoulder, the main edge, and the postedge shoulder.

theoretical spectra sampled over AIMD simulations of the supercell models depicted in Figure 1. As a first approximation, the experimental spectra look rather similar to each other with a maximum at about $404.3 \mathrm{eV}$ and a similar shape. However, a closer inspection indicates that the MAPI spectrum is shifted by $-0.23 \mathrm{eV}$ in comparison to the MAPB spectrum, and it has a less pronounced shoulder at about $406 \mathrm{eV}$. Moreover, we note that the resonance at $415 \mathrm{eV}$ seen in the extended range of the spectra belongs to the $\mathrm{Pb} \mathrm{N} \mathrm{N}_{5}$ edge ( $\mathrm{Pb} 4 \mathrm{~d}$-to- $6 \mathrm{p}$ *; see Figures S2 and S3) and that the spectra also indicate some differences here. The shape of the experimental K-edge of MAPI resembles previous measurements. ${ }^{15}$ The primary aim of the simulations is to rationalize the differences in the N K-edge main edge positions of MAPI and MAPB and assign their general features.

The theoretical N 1s XA spectra for MAPI and MAPB in Figure 2 are averaged over all nitrogens among all sampled geometries. The two calculated spectra show the same characteristics: a pre-edge shoulder, a main edge absorption peak, and a postedge shoulder. The peak maxima of the theoretical spectra were aligned to the experimental MAPI spectrum, and the MAPB spectrum was shifted by the same energy. Because the calculated spectra do not reproduce the absolute excitation energies, we refer instead to the relative MAPI/MAPB shift which remains unchanged by shifting. The shift is performed to make the direct comparison between the calculated and experimental spectra more straightforward in the figure and is aligned to MAPI because those are the figures shown in the main body of this work. The maximum of the main peak intensity between MAPI and MAPB for the theoretical spectra is shifted by $0.31 \mathrm{eV}$, in good agreement with the experiment. Further shifting of the calculated spectra relative to each other such that their peak intensities align 
shows that they largely overlap in shape, indicating a nearly systematic shift between the two systems; this is shown in Figure S4. However, for the calculated MAPB spectrum, we observe a small increase in the postedge shoulder, $2 \mathrm{eV}$ above the peak maxima, in agreement with the experimental findings.

Although the difference of the main peak intensity in the experimental and calculated XA spectra of MAPI and MAPB is well reproduced, the calculated spectra are much too narrow and the shapes and differences in the postedge region are not captured. This can be improved with a larger broadening, but consequently, the spectral detail (in particular the pre-edge shoulder) is reduced. The performance of the transition potential approximation has been evaluated before in N 1s XA spectra of ammonium species ${ }^{12,13}$ and in which similar artifacts related to the transition potential approximation have been observed. Thus, we probe the sensitivity in the calculated spectra to geometric variation only around the pre- and mainedge features.

To show that the sampling of configurations selected for spectrum geometries for MAPI and MAPB are representative of the AIMD trajectory, we compare the radial distribution functions (RDFs) of the sampled geometries and the whole simulation. A plot of this is shown in Figure 3 for the $\mathrm{H} \cdots \mathrm{X}$ distance, and the close agreement between the MAPI and MAPB RDFs demonstrates that the five sampled geometries provide a good representation of both systems.

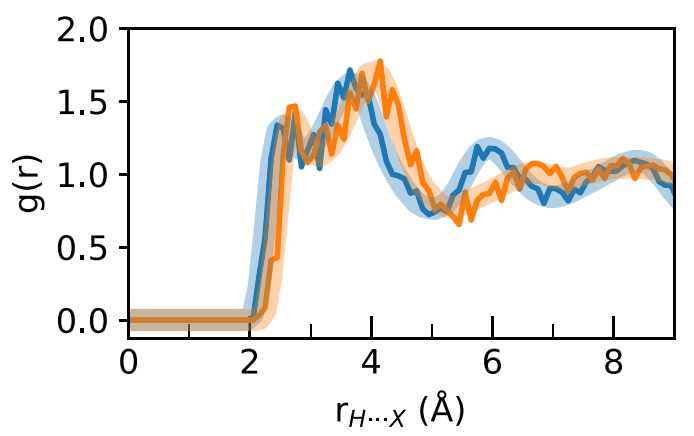

Figure 3. $\mathrm{RDF}$ of $\mathrm{H} \cdots \mathrm{I}$ (orange) and $\mathrm{H} \cdots \mathrm{Br}$ (blue) distances for nitrogen-attached hydrogens during the full simulation (transparent lines) and the sampled geometries (solid lines). For both systems, the good agreement validates the sampling as representative of the whole simulation.

However, due to the systems having different geometries and different crystal structures (tetragonal MAPI vs cubic MAPB), it is unclear whether the $-0.3 \mathrm{eV}$ spectral shift, observed in Figure 2, is due to a chemical difference between iodine and bromine or the geometric differences of the systems. To investigate the sensitivity to the structure, we took one configuration of MAPI and replaced all iodines with bromines to create an identical tetragonal MAPB and recalculated the XA spectrum. The result of this is shown in Figure 4. The resulting MAPI to MAPB peak shift at a common geometry is $0.29 \mathrm{eV}$, essentially the same as the shift for the aggregate sampled spectra. The MAPB spectrum also has a slightly more prominent postedge shoulder than in Figure 2, but this is not unusual compared to some of the other individual spectra. We notice that the artificially long distances around $\mathrm{Br}$ seem to influence the intensity of the spectrum. However, this clearly indicates that the peak intensity shift is not due to differences in the geometries of the systems but

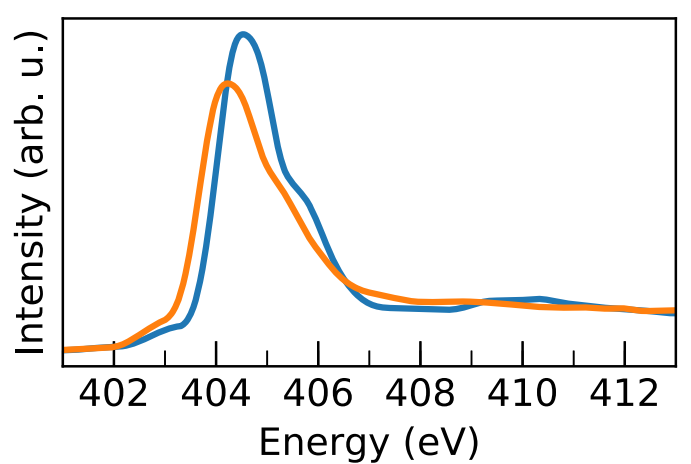

Figure 4. Comparison of $\mathrm{N}$ K-edge spectra for a MAPI configuration (orange) and the same configuration with I replaced by $\mathrm{Br}$ (blue). Peak maxima occur at 404.23 and $404.52 \mathrm{eV}$. This peak-intensity shift of $0.29 \mathrm{eV}$ matches the shift from Figure $2 \mathrm{of} 0.31 \mathrm{eV}$, indicating that the shift is not mainly due to the cell structure.

rather an electronic difference between the halides. We also notice that the differences in band gap between the two systems-1.55 eV for MAPI ${ }^{29,30}$ and $2.28 \mathrm{eV}$ for MAPB, ${ }^{10} \mathrm{a}$ difference of $0.73 \mathrm{eV}$-is twice the shift in the absorption energies. An additional comparison is done between the PBE and BLYP functionals (Figure S5) to investigate the functional effects, but these functionals showed similar MAPI-MAPB shifts and spectral features.

The experimental spectrum is an average of many different nitrogens in various accessible local environments; therefore, in order to effectively compare theoretical results to it, we similarly need to simulate XA spectra for a range of nitrogens in different local environments. Each nitrogen site in each sampled AIMD configuration requires a separate $\mathrm{N}$ 1s XA spectrum calculation. A total of 160 individual $\mathrm{N}$ spectra were calculated for MAPI and 180 for MAPB. Averaged together, these spectra are then meaningfully compared to experimental results, as shown in Figure 2.

However, instead of simply averaging all spectra together, we can organize the spectra into "sub-averages" according to some criterion to see how that affects the spectral shape and features. This can be done by choosing some metric (e.g., N-C distance), ordering the spectra accordingly, and averaging them into blocks for a fixed distance range. The averaging aims to smooth out any other effects to isolate only the effect of the coordinate we are interested in under the assumption that they are not strongly correlated. This averaging procedure is, however, vulnerable to situations where a block may have only one or two spectra in it. These blocks are not well-averaged and therefore cannot properly be compared to the other wellaveraged blocks in terms of trends along the coordinate, and thus they are not shown in the block average comparison figures.

The distance block averaging is illustrated graphically in Figure 5 for the $\mathrm{N}-\mathrm{C}$ distance in MAPB. For each nitrogen, the spectrum is plotted as a thin line and offset vertically according to its N-C distance, as shown on the left axis. These are then split into $0.05 \AA$ blocks, and all spectra in each block are averaged, represented by the thick black spectral lines. It is these thick black spectral lines that provide the appropriate information of interest regarding the averaged effect of the coordinate on the spectra, so in the presentation below, we report only those averages, centered on the same starting point to better compare relative changes among the spectra. 


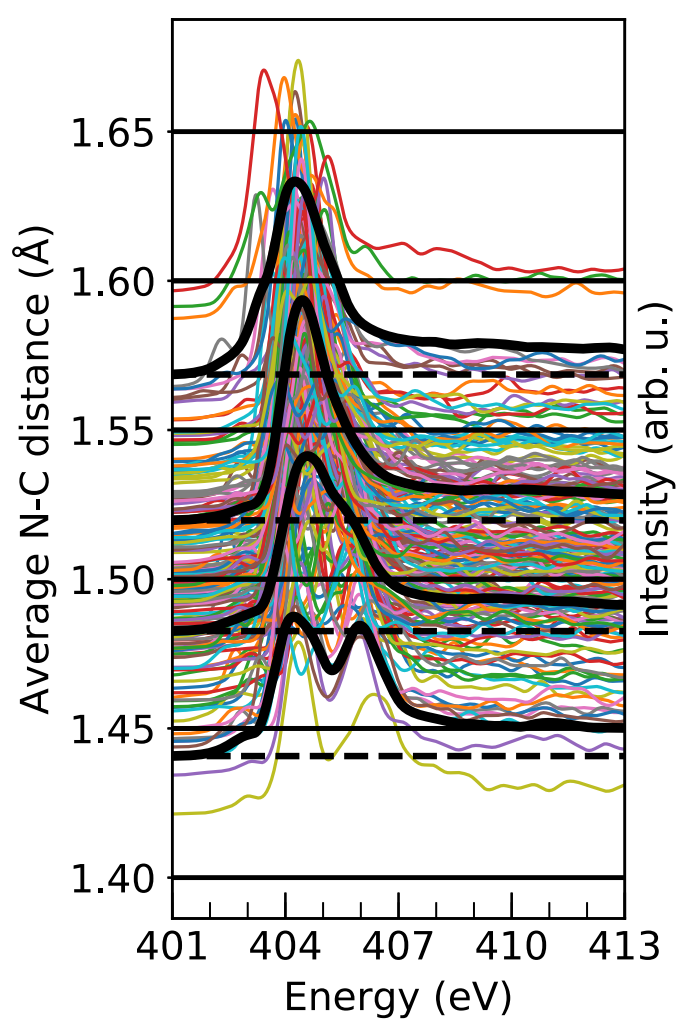

Figure 5. All individual $\mathrm{N}$ 1s XA spectra for the sampled MAPB geometries (thin lines) sorted by the $\mathrm{N}-\mathrm{C}$ distance for each respective nitrogen. The $0.05 \AA$ blocks are delineated with horizontal black lines, and the block spectra averages are shown as thick black lines. The block averages are reproduced in Figure S6.

Since we are interested in geometric effects on the nitrogen XA spectrum, our focus is on coordinates that involve the $\mathrm{MA}^{+}$ molecular ion. First, we report the intramolecular coordinates, $\mathrm{N}-\mathrm{C}$ distance, and average $\mathrm{N}-\mathrm{H}$ distance. Starting with the $\mathrm{N}-\mathrm{C}$ coordinate, as shown in Figures 6 and S6, a clear trend is

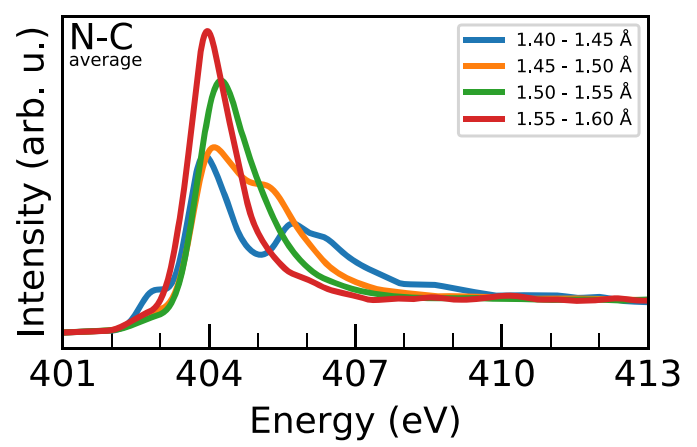

Figure 6. Block-averaged MAPI N 1s XA spectra ordered by N-C distance in $0.05 \AA$ blocks from 1.40 to $1.60 \AA$. Explanation of the trends is given in the text, and the block-averaging procedure is given in Figure 5 .

seen for both MAPI and MAPB that a decrease in the N-C distance gives rise to a splitting of the main peak into two peaks; one which stays around $404 \mathrm{eV}$ and the other which progressively blue shifts. The average sampled $\mathrm{N}-\mathrm{C}$ bond lengths of MAPI and MAPB are both $1.50 \AA$, which explains the slight postedge shoulder seen in Figure 2 as a combination of orange and green lines. The dependence on the $\mathrm{N}-\mathrm{C}$ distance is clearly shown, but it is not a degree of freedom changing between MAPI and MAPB.

Next, we turn to analyzing the other pertinent intramolecular distance coordinate, the average $\mathrm{N}-\mathrm{H}$ distance for the hydrogens attached to each nitrogen. These data are shown in Figures 7 and S7. One notable trend that these plots show is

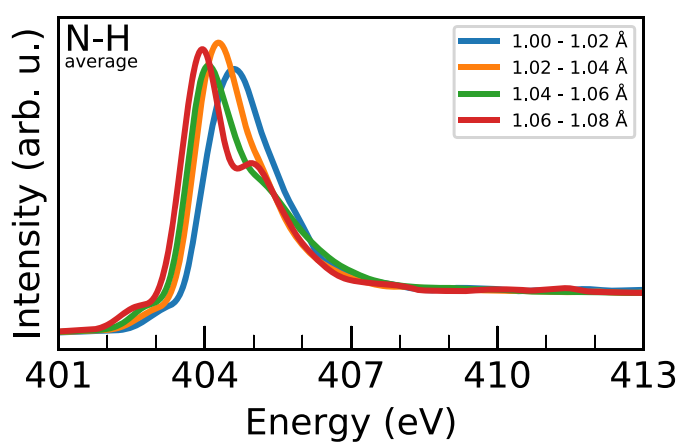

Figure 7. Block-averaged MAPI N 1s XA spectra ordered by the average $\mathrm{N}-\mathrm{H}$ distance in $0.02 \AA$ blocks from 1.00 to $1.08 \AA$. Explanation of the trends is given in the text, and the block-averaging procedure is given in Figure 5.

that the longer $\mathrm{N}-\mathrm{H}$ distances seem to exhibit more of the postedge shoulder behavior associated with the short $\mathrm{N}-\mathrm{C}$ distances shown previously. An analysis of the sampled structures does show a slight inverse relationship between these coordinates, as shown in Figure S8. Specific to just the $\mathrm{N}-\mathrm{H}$ distances, however, the plots show that increasing $\mathrm{N}-\mathrm{H}$ distance is associated with a red-shifting of the whole spectrum, particularly when examining the main peak positions. As with the $\mathrm{N}-\mathrm{C}$ analysis, this is borne out in the combined average spectra. The average $\mathrm{N}-\mathrm{H}$ distance for both systems is $1.04 \AA$, which is between the orange and green plots. For MAPI, these two peaks average to a maximum intensity at $404.18 \mathrm{eV}$, and for MAPB, they average to $404.49 \mathrm{eV}$, comparing favorably to the total averages of 404.2 and 404.47 $\mathrm{eV}$, respectively. In analogy with previous studies of ethylamines, ${ }^{13}$ the trends in the distance dependence in Figures 6 and 7 can be understood in terms of shape resonances for the antibonding $\sigma_{\mathrm{N}-\mathrm{C}^{*}}$ and $\sigma_{\mathrm{N}-\mathrm{H}^{*}}$ orbitals, and the splitting of the $\mathrm{XA}$ spectrum into a main-edge and a separate postedge figure can be rationalized by the anticorrelation of the $\mathrm{N}-\mathrm{C}$ and $\mathrm{N}-$ $\mathrm{H}$ distances observed in Figure S8.

As stated in the introduction, based on previous work by Ekimova et al., ${ }^{12}$ we believe hydrogen bonding between the nitrogen-attached hydrogens and the lattice $\mathrm{I} / \mathrm{Br}$ to be important. Specifically, based on their results, it is expected that increasing $\mathrm{N}-\mathrm{H}$ hydrogen bond donation will cause a broadening of the spectrum. Given that there is a hydrogen bonding effect on the spectrum, one naturally would expect to see some difference between a nitrogen with all hydrogens strongly donating and one with no hydrogens donating. However, it would also be expected to be possible to distinguish between symmetrically donating nitrogens and asymmetrically donating nitrogens. To investigate this, we order the spectra by the difference between the shortest and longest $\mathrm{H} \cdots \mathrm{I}$ or $\mathrm{H} \cdots \mathrm{Br}$ distances (Figures 8 and S9, respectively) for each nitrogen; a lower value indicates symmetry, while a higher value indicates asymmetry, though this does not necessarily give information about the strength or number of hydrogen-bonding interactions. The results of this 


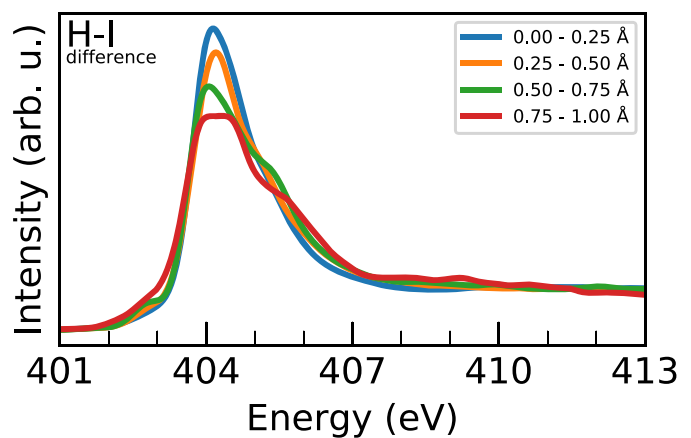

Figure 8. Block-averaged MAPI N 1s XA spectra ordered by the difference in the shortest and longest nitrogen $\mathrm{H} \cdots \mathrm{I}$ distance in $0.25 \AA$ blocks from 0 to $1 \AA$. Explanation of the trends is given in the text, and the block-averaging procedure is given in Figure 5.

analysis indicate that there is a clear effect of this asymmetry on the peak intensity and broadening of the spectrum. Additionally, in contrast to previous coordinates, this asymmetry also correlates with the strength of the pre-edge shoulder. This is also in line with the previous results of Ekimova et al., who argued that the appearance of an ammonia pre-edge was due to differences in the symmetry of the lowest unoccupied molecular orbital (LUMO) as compared to the symmetric ammonium which had no pre-edge and that the slight pre-edge for aqueous ammonium is related to instantaneous distortions of symmetry. ${ }^{13}$

To complement the block-averaging analysis and gain a deeper understanding of the correlation between the spectra and investigated geometric coordinates, we also carried out a detailed analysis of molecular orbitals involved in the prominent transitions and the corresponding unoccupied partial density of states (PDOS). For this purpose, we have chosen two specific configurations corresponding to methylammonium in MAPI with relatively shorter (1.475 $\AA$ ) and longer $(1.497 \AA) \mathrm{N}-\mathrm{C}$ bond distances. In the former configuration, there is also a large pre-edge intensity associated with one very long nitrogen $\mathrm{H} \cdots \mathrm{I}$ distance and a large asymmetry $\left(\mathrm{H}_{1} \cdots \mathrm{I}=3.48 \AA, \mathrm{H}_{2} \cdots \mathrm{I}=2.82 \AA\right.$, and $\mathrm{H}_{3} \cdots \mathrm{I}=2.65$ $\AA$; asymmetry $=0.83 \AA$ ), whereas in the other configuration with an equally large asymmetry, there is instead one very short nitrogen $\mathrm{H} \cdots \mathrm{I}$ distance $\left(\mathrm{H}_{1} \cdots \mathrm{I}=3.17 \AA \mathrm{H}_{2} \cdots \mathrm{I}=2.91 \AA\right.$, and $\mathrm{H}_{3} \cdots \mathrm{I}=2.37 \AA$; asymmetry $=0.80 \AA$ ). Therefore, we have selected configurations that allow us to investigate the mechanisms for spectral response both to changes in the $\mathrm{N}-$ $\mathrm{C}$ bond distance, as presented in Figure 6, and to variations in hydrogen bonding, as presented in Figures 7 and 8 .

The results of the orbital analysis are presented in Figures 9 and 10. These figures also contain molecular orbitals around $\mathrm{MA}^{+}$from the strong transitions in pre-, main-, and postedge regions in the TP_HH calculations and corresponding PDOS of carbon and nitrogen in the core-excited $\mathrm{MA}^{+}$ion. For reference, the ground-state Kohn-Sham orbitals of an isolated $\mathrm{MA}^{+}$ion are given in Figure S10. It should be noted that the $a_{1}$ and $e$ symmetries correspond to the $\sigma$ and $\pi$ character along the $\mathrm{N}-\mathrm{C}$ bond.

We note that the intensity of spectra readily follows the PDOS of $\mathrm{N} \mathrm{p}$ orbitals, but the XA intensity is more pronounced near the edge where the $N 2 p$ contribution dominates. The results of the PDOS in Figures 9 and 10 clearly indicate that the states in the pre-edge region for both cases have major contributions from $\mathrm{N} \mathrm{s}, \mathrm{N} \mathrm{p}$, and $\mathrm{C}$ p orbitals. They

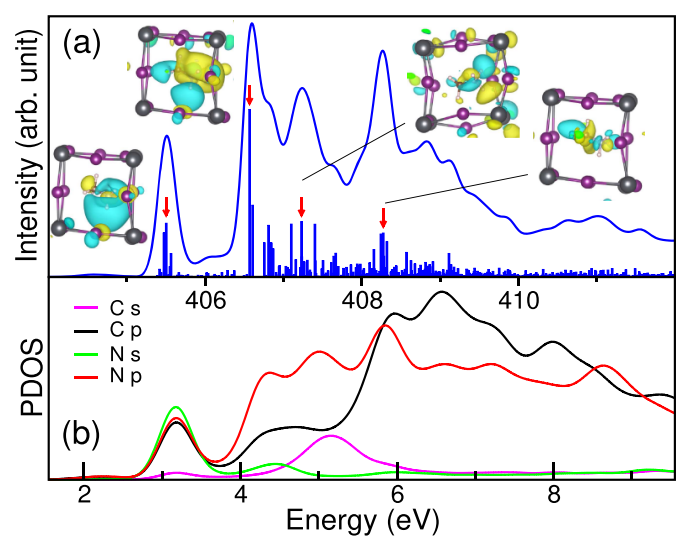

Figure 9. (a) Individual $\mathrm{N}$ 1s XA spectrum (line) for a specific $\mathrm{N}$ atom in MAPI with a shorter $\mathrm{N}-\mathrm{C}$ distance. The molecular orbitals (bars) around the $\mathrm{MA}^{+}$ion are given, and the specific ones corresponding to the inlaid images are indicated by red arrows. (b) Corresponding unoccupied PDOS for the system.

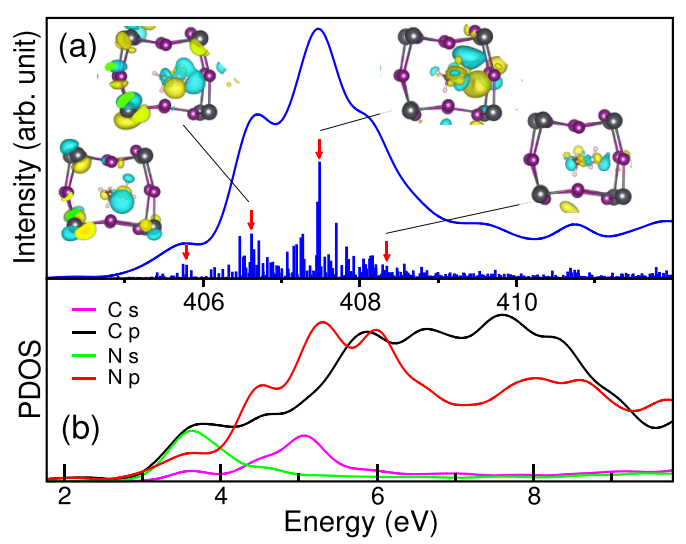

Figure 10. a) Individual $\mathrm{N}$ 1s XA spectrum (line) for a specific $\mathrm{N}$ atom in MAPI with a longer $\mathrm{N}-\mathrm{C}$ distance. The molecular orbitals (bars) around the $\mathrm{MA}^{+}$ion are given, and the specific ones corresponding to the inlaid images are indicated by red arrows. (b) Corresponding unoccupied PDOS for the system.

possess the character of the LUMO, with $6 a_{1}$ symmetry, of the isolated $\mathrm{MA}^{+}$ion in Figure S10. However, depending on the hydrogen bond environment, the cyan orbital lobe around nitrogen has been strongly distorted due to the interaction of the $\mathrm{MA}^{+}$ion with the surrounding $\mathrm{Pb}-\mathrm{I}$ cage.

For the configuration in Figure 9, there is sufficient room for a large lobe of the $6 a_{1}$ orbital to form, whereas in Figure 10, the strong confinement around the ammonium group results in a significant quenching of the orbital amplitude. Due to this, the $\mathrm{N} p$ character in the states at the pre-edge region has been significantly reduced as seen in the PDOS. In both cases, we observe a localization of the $6 a_{1}$ lobe in the direction of the longest $\mathrm{H} \cdots \mathrm{I}$ hydrogen bond and a hybridization with a small amount of I $5 \mathrm{p}$ to form antibonding $\sigma^{*}(\mathrm{H} \cdots \mathrm{I})$ hydrogen bonding character. As noted in the block averaging in Figure 8, the intensity in the pre-edge region is enhanced by asymmetry, which increases the $\mathrm{N} \mathrm{p}$ character in the transition as seen in the $\mathrm{N}$ p PDOS, and here we note that predominantly long hydrogen bonds contribute to the pre-edge feature as observed in liquid water. ${ }^{31,32}$

The transitions identified as contributing to the second $(406.6 \mathrm{eV})$ and third $(407.2 \mathrm{eV})$ features have more mixed characters but resemble the LUMO + $1(3 e)$ and LUMO + 3 
(4e) orbitals of the isolated $\mathrm{MA}^{+}$ion, both having $\pi(\mathrm{N}-\mathrm{C})$ symmetry and antibonding $\sigma^{*}(\mathrm{~N}-\mathrm{H})$ character. The strong feature at $408.3 \mathrm{eV}$ in the postedge region in Figure 9 is due to states which have significant contributions from antibonding $\sigma^{*}(\mathrm{~N}-\mathrm{C})$ character associated with orbital $8 a_{1}$, pushed up in energy by the short $\mathrm{N}-\mathrm{C}$ distance, and appears much less pronounced in Figure 10. The molecular states $\left(7 a_{1}\right.$ and $\left.9 a_{1}\right)$ with strong $\mathrm{C} s$ and $\mathrm{C} \mathrm{p}_{z}$ (along $\mathrm{N}-\mathrm{C}$ bond) characters have not contributed significantly to any prominent peaks. The analysis indicates that the main peak intensity corresponds to states with the $\pi(\mathrm{N}-\mathrm{C})$ symmetry and antibonding $\sigma^{*}(\mathrm{~N}-\mathrm{H})$ character but with contributions of states with $\sigma^{*}(\mathrm{~N}-\mathrm{C})$ orbital character for longer $\mathrm{N}-\mathrm{C}$ distance, which is redistributed to the postedge peak at a reduced $\mathrm{N}-\mathrm{C}$ distance.

In addition to the previously mentioned fact that the MAPI and MAPB simulations used different crystal structures at 300 $\mathrm{K}$ (tetragonal and cubic, respectively), the fact that bromine is a smaller atom than iodine means the inorganic lattice grid in the material is smaller, and that will affect any direct comparison of MA-lattice distances between the two systems. The RDFs for $\mathrm{N}-\mathrm{I} / \mathrm{Br}, \mathrm{H}-\mathrm{Pb}$ (for nitrogen hydrogens), and $\mathrm{N}-\mathrm{Pb}$ are shown in the Supporting Information in Figures S11-S13. In short, these RDFs appear to show similar distributions for both systems when accounting for the difference in ion sizes (i.e., relative peak behavior).

The $\mathrm{H} \cdots \mathrm{I} / \mathrm{Br}$ (for nitrogen-attached hydrogens) RDFs, in contrast, show qualitatively different long-range behavior between the two systems, as seen in Figure 11. Vertical lines

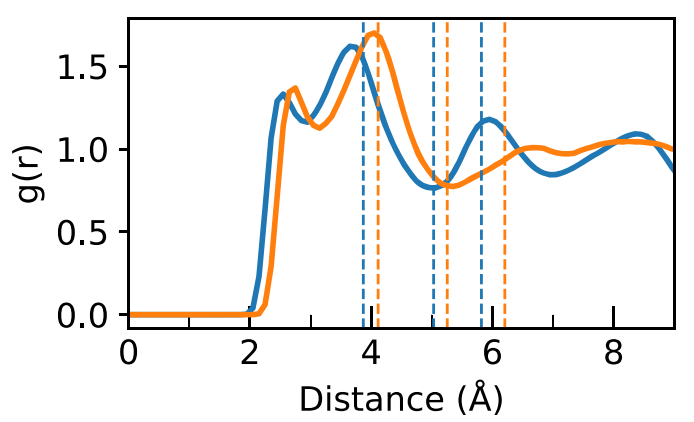

Figure 11. RDF of $\mathrm{H} \cdots \mathrm{Br}$ (blue) and $\mathrm{H} \cdots \mathrm{I}$ (orange) distances for nitrogen-attached hydrogens during the simulations of MAPB and MAPI. The distances with an integral of 4,8 , and 12 , corresponding to fractions of the $\mathrm{Pb}-\mathrm{I} / \mathrm{Br}$ cage for each $\mathrm{MA}^{+}$molecular ion, for each $\mathrm{RDF}$ are shown in their respective colors from left to right.

are drawn for each RDF corresponding to an integral value of 4,8 , and 12 , from left to right, corresponding to fractions of the 12 -atom $\mathrm{Pb}-\mathrm{I} / \mathrm{Br}$ cage that each $\mathrm{MA}^{+}$molecular ion is in. For short distances ( $<5 \AA$ ), the RDFs look similar except for a slight expected lengthening effect for MAPI, but for long distances ( $>5 \AA$ ), it is seen that MAPB continues to have more distinct peaks as compared to MAPI which is much more smoothed out in its distribution.

To determine if this effect was due to differences in the crystal structure or supercells, which are somewhat constrained in the simulation, we took the end of the MAPI simulation and replaced all iodines with bromines to make an identical tetragonal MAPB system. This system was rescaled to the experimental tetragonal $\beta$-MAPB parameters from Poglitsch and Weber $^{27}$ and run in an NPT MD simulation as before for another $10 \mathrm{ps}$. Two separate simulations were performed, one at $300 \mathrm{~K}$ as before and another at $200 \mathrm{~K}$ because the tetragonal-cubic transition in MAPB occurs at $237 \mathrm{~K}$. The RDFs for these short simulations were then compared to the previous RDFs, as shown in Figure 12. In this figure, it can be

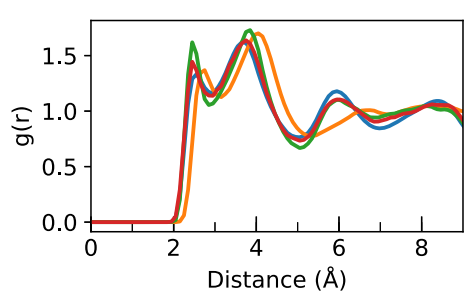

Figure 12. RDF of $\mathrm{H} \cdots \mathrm{Br}$ (blue) and $\mathrm{H} \cdots \mathrm{I}$ (orange) distances for nitrogen-attached hydrogens during the 50 ps simulations of cubic $\mathrm{MAPB}$ and tetragonal MAPI are compared to tetragonal MAPB RDFs after an extra $10 \mathrm{ps}$ of simulation at $200 \mathrm{~K}$ (green) or $300 \mathrm{~K}$ (red). It can be seen that both tetragonal MAPB RDFs match the cubic MAPB $\mathrm{RDF}$ much more than the tetragonal MAPI RDF.

seen that there is almost no difference between the 200 and $300 \mathrm{~K}$ simulations and that both of these simulations match up very well with the cubic MAPB RDF. Furthermore, the tetragonal MAPB spectra at both 200 and $300 \mathrm{~K}$ (Figures S14 and $S 15$, respectively) are very similar to the original cubic MAPB spectrum, not the tetragonal MAPI spectrum. This indicates that the long-range difference observed here is not due to crystal structure differences but instead due to the difference in atomic radii between the two halide ions.

To summarize the Results section, careful measurements of nitrogen K-edge XA spectra of MAPI and MAPB show distinct spectral differences which we have dissected theoretically by a combination of AIMD simulations and spectrum simulations on the two compounds. The combination allows us to determine aspects of the spectra related to structural changes and to purely electronic changes from halide substitution.

\section{CONCLUSIONS}

In a combined experimental and theoretical study, we compared the N 1s XA spectra of room-temperature structures of tetragonal MAPI and cubic MAPB. The experimental data, coming from measurements on single crystals in UHV, allow for clean N 1s XA spectra largely free from beam damage and a detailed comparison of MAPI and MAPB. Theoretical models compare favorably with the experimental spectra. We observe an approximately $0.3 \mathrm{eV}$ blue shift of the MAPB spectrum relative to the MAPI spectrum, which is not due to the crystal structure differences but due to the difference in band gaps, indicating that electronic structure information is encoded in $\mathrm{N}$ 1s XAS.

AIMD simulations were performed and sampled for the XA spectrum analysis. In order to gain a more detailed understanding of the $\mathrm{N}$ 1s XA spectra of hybrid perovskites, the individual nitrogen spectra were then ordered and averaged along various intra- and intermolecular coordinates relative to methylammonium counterion atoms-specifically the $\mathrm{N}-\mathrm{C}$ bond length, average $\mathrm{N}-\mathrm{H}$ length, and $\mathrm{H} \cdots \mathrm{I} / \mathrm{Br}$ asymmetryto determine the effects of these distances on the XA spectra. It was found that a shorter $\mathrm{N}-\mathrm{C}$ length corresponds to the appearance of a higher-energy peak that leads to a postedge shoulder feature in the overall spectrum, and a shorter $\mathrm{N}-\mathrm{H}$ length corresponds to blue-shifting the entire spectrum, which is also borne out in the overall calculated spectrum. It was also shown that the $\mathrm{H} \cdots \mathrm{I} / \mathrm{Br}$ asymmetry corresponds to a decrease 
in the main peak intensity and the appearance of the pre-edge shoulder. These trends are generally clearer in the MAPI spectra but are also represented well in the MAPB spectra shown in the Supporting Information.

Finally, it is reported that the long-range behavior of the $\mathrm{H}$... $\mathrm{I} / \mathrm{Br}$ distances are different between MAPI and MAPB according to their RDFs. This effect holds even when accounting for the differing crystal structures by performing a tetragonal MAPB simulation, and we attribute it to the difference in atomic radii.

These results provide information on how the local structure of the material can be seen from its effects on the XA spectra. In particular, it points to the role of hydrogen bonding (asymmetry) in the spectrum, and thus the electronic structure, which can be important for further development of hybrid perovskite materials for solar cell applications.

\section{ASSOCIATED CONTENT}

\section{SI Supporting Information}

The Supporting Information is available free of charge at https://pubs.acs.org/doi/10.1021/acs.jpcc.1c02017.

Extra calculated XA spectrum comparison plots, the MAPB block-averaged plots, additional RDFs, isolated $\mathrm{MA}^{+}$orbitals, and a comparison of experimental methods for the MAPI spectrum (PDF)

\section{AUTHOR INFORMATION}

\section{Corresponding Author}

Michael Odelius - Department of Physics, Stockholm University, SE-106 91 Stockholm, Sweden; 다이.org/ 0000-0002-7023-2486; Email: odelius@fysik.su.se

\section{Authors}

Cody M. Sterling - Department of Physics, Stockholm University, SE-106 91 Stockholm, Sweden; (1) orcid.org/ 0000-0001-9518-9405

Chinnathambi Kamal - Department of Physics, Stockholm University, SE-106 91 Stockholm, Sweden; Theory and Simulations Laboratory, HRDS, Raja Ramanna Centre for Advanced Technology, 452013 Indore, India; (1) orcid.org/ 0000-0002-4546-8219

Gabriel J. Man - Department of Physics and Astronomy, Uppsala University, SE-751 20 Uppsala, Sweden; (1) orcid.org/0000-0003-2932-7018

Pabitra K. Nayak - TIFR Centre for Interdisciplinary Sciences, Tata Institute of Fundamental Research, 500046 Hyderabad, India; (1) orcid.org/0000-0002-7845-5318

Konstantin A. Simonov - Department of Physics and Astronomy, Uppsala University, SE-751 20 Uppsala, Sweden

Sebastian Svanström - Department of Physics and Astronomy, Uppsala University, SE-751 20 Uppsala, Sweden; (1) orcid.org/0000-0001-7351-8183

Alberto García-Fernández - Division of Applied Physical Chemistry, Department of Chemistry, KTH Royal Institute of Technology, SE-100 44 Stockholm, Sweden; (O) orcid.org/ 0000-0003-1671-9979

Thomas Huthwelker - Swiss Light Source, Paul Scherrer Institut, 5232 Villigen, Switzerland

Ute B. Cappel - Division of Applied Physical Chemistry, Department of Chemistry, KTH Royal Institute of Technology, SE-100 44 Stockholm, Sweden; 이이.org/ 0000-0002-9432-3112
Sergei M. Butorin - Department of Physics and Astronomy, Uppsala University, SE-751 20 Uppsala, Sweden; (1) orcid.org/0000-0003-3242-5305

Håkan Rensmo - Department of Physics and Astronomy, Uppsala University, SE-751 20 Uppsala, Sweden

Complete contact information is available at:

https://pubs.acs.org/10.1021/acs.jpcc.1c02017

\section{Notes}

The authors declare no competing financial interest.

\section{ACKNOWLEDGMENTS}

C.M.S., C.K., and M.O. acknowledge funding from the Swedish Research Council (VR contract 2015-03956 and 2016-04590), the European Union's Horizon 2020 research and innovation programme under the Marie Skłodowska-Curie grant agreement no. 860553, and the Swedish Energy Agency (contract 2017-006797). The calculations were enabled by resources provided by the Swedish National Infrastructure for Computing (SNIC) at the Swedish National Supercomputer Center (NSC), the High Performance Computer Center North (HPC2N), and Chalmers Centre for Computational Science and Engineering (C3SE), partially funded by the Swedish Research Council through grant agreement 201805973. H.R., G.J.M., and S.S. acknowledge the Swedish Research Council (grants 2018-06465 and 2018-04330), the Swedish Foundation for Strategic Research (RMA15-0130), and the Swedish Energy Agency (grant P43549-1) for funding. U.B.C. and A.G.F. acknowledge funding from the Carl Tryggers foundation and the Göran Gustafssons foundation. P.K.N. acknowledges support from the Department of Atomic Energy, Government of India, under Project Identification no. RTI 4007 and SERB India core research grant (CRG/2020/ 003877). S.M.B. acknowledges support from the Swedish Research Council (research grant 2018-05525). The authors thank Erika Giangrisostomi and Ruslan Ovsyannikov for support at the PM4 beamline and Helmholtz-Zentrum Berlin für Materialien und Energie for the allocation of beamtime at PM4 (191-08328, 192-08712, and 192-08527). The research leading to this result has been supported by the project CALIPSOplus under the grant agreement 730872 from the EU Framework Programme for Research and Innovation HORIZON 2020. They acknowledge MAX IV Laboratory for time on Beamline FlexPES under proposal 20200451. Research conducted at MAX IV, a Swedish national user facility, is supported by the Swedish Research Council under contract 2018-07152, the Swedish Governmental Agency for Innovation Systems under contract 2018-04969, and Formas under contract 2019-02496. They also thank Stephan Appelfeller, Alexei Preobrajenski, and Alexander Generalov for support during the FlexPES beamtime. They thank the Paul Scherrer Institute for providing beamtime at the PHOENIX beamline.

\section{REFERENCES}

(1) Hou, X.; Wang, X.; Mi, W.; Du, Z. Prediction on electronic structure of $\mathrm{CH}_{3} \mathrm{NH}_{3} \mathrm{PbI}_{3} / \mathrm{Fe}_{3} \mathrm{O}_{4}$ interfaces. Solid State Commun. 2018, 269, 90-95.

(2) Lindblad, R.; Bi, D.; Park, B.-w.; Oscarsson, J.; Gorgoi, M.; Siegbahn, H.; Odelius, M.; Johansson, E. M. J.; Rensmo, H. Electronic Structure of $\mathrm{TiO}_{2} / \mathrm{CH}_{3} \mathrm{NH}_{3} \mathrm{PbI}_{3}$ Perovskite Solar Cell Interfaces. J. Phys. Chem. Lett. 2014, 5, 648-653. 
(3) Kot, M.; Wojciechowski, K.; Snaith, H.; Schmeisser, D. Evidence of Nitrogen Contribution to the Electronic Structure of the $\mathrm{CH}_{3} \mathrm{NH}_{3} \mathrm{PbI}_{3}$ Perovskite. Chem.-Eur. J. 2018, 24, 3539-3544.

(4) Lindblad, R.; Jena, N. K.; Philippe, B.; Oscarsson, J.; Bi, D.; Lindblad, A.; Mandal, S.; Pal, B.; Sarma, D. D.; Karis, O.; et al. Electronic Structure of $\mathrm{CH}_{3} \mathrm{NH}_{3} \mathrm{PbX}_{3}$ Perovskites: Dependence on the Halide Moiety. J. Phys. Chem. C 2015, 119, 1818-1825.

(5) Shen, P.; Nie, K.; Sun, X.; Liu, L.; McLeod, J. A. Electronic structure of formamidinium ions in lead triiodide perovskites. Phys. Status Solidi RRL 2016, 10, 677-681.

(6) Wang, M.; Sheng, C.-X.; Zhang, C.; Yao, J. Emissive edge state in $\mathrm{CH}_{3} \mathrm{NH}_{3} \mathrm{PbBr}_{3}$ films probed by fluorescence lifetime imaging technique. J. Photonics Energy 2018, 8, 1.

(7) Jena, A. K.; Kulkarni, A.; Miyasaka, T. Halide Perovskite Photovoltaics: Background, Status, and Future Prospects. Chem. Rev. 2019, 119, 3036-3103.

(8) National Renewable Energy Laboratory: Best Research-Cell Efficiencies. Accessed 15 April 2020. https://www.nrel.gov/pv/ assets/pdfs/best-research-cell-efficiencies.20200406.pdf.

(9) Herz, L. M. How Lattice Dynamics Moderate the Electronic Properties of Metal-Halide Perovskites. J. Phys. Chem. Lett. 2018, 9, 6853-6863.

(10) Wang, L.; Yuan, G. D.; Duan, R. F.; Huang, F.; Wei, T. B.; Liu, Z. Q.; Wang, J. X.; Li, J. M. Tunable bandgap in hybrid perovskite $\mathrm{CH}_{3} \mathrm{NH}_{3} \mathrm{~Pb}\left(\mathrm{Br}_{3-y} X_{y}\right)$ single crystals and photodetector applications. AIP Adv. 2016, 6, 045115 .

(11) Svane, K. L.; Forse, A. C.; Grey, C. P.; Kieslich, G.; Cheetham, A. K.; Walsh, A.; Butler, K. T. How Strong Is the Hydrogen Bond in Hybrid Perovskites? J. Phys. Chem. Lett. 2017, 8, 6154-6159.

(12) Ekimova, M.; Quevedo, W.; Szyc, Ł.; Iannuzzi, M.; Wernet, P.; Odelius, M.; Nibbering, E. T. J. Aqueous Solvation of Ammonia and Ammonium: Probing Hydrogen Bond Motifs with FT-IR and Soft Xray Spectroscopy. J. Am. Chem. Soc. 2017, 139, 12773-12783.

(13) Ekimova, M.; Kubin, M.; Ochmann, M.; Ludwig, J.; Huse, N.; Wernet, P.; Odelius, M.; Nibbering, E. T. J. Soft X-ray Spectroscopy of the Amine Group: Hydrogen Bond Motifs in Alkylamine/ Alkylammonium Acid Base Pairs. J. Phys. Chem. B 2018, 122, 7737-7746.

(14) Drisdell, W. S.; Leppert, L.; Sutter-Fella, C. M.; Liang, Y.; Li, Y.; Ngo, Q. P.; Wan, L. F.; Gul, S.; Kroll, T.; Sokaras, D.; et al. Determining Atomic-Scale Structure and Composition of OrganoLead Halide Perovskites by Combining High-Resolution X-ray Absorption Spectroscopy and First-Principles Calculations. ACS Energy Lett. 2017, 2, 1183-1189.

(15) Man, G. J.; Sterling, C.; Kamal, C.; Simonov, K. A.; Svanström, S.; Acharya, J.; Johansson, F. O. L.; Giangrisostomi, E.; Ovsyannikov, R.; Huthwelker, T. et al. Electronic Coupling between the Organic and Inorganic Sub-Lattices of a Hybrid Organic-Inorganic Perovskite Single Crystal. 2020, arXiv:2011.02016 [cond-mat].

(16) Tanuma, S.; Powell, C. J.; Penn, D. R. Calculations of electron inelastic mean free paths. V. Data for 14 organic compounds over the 50-2000 eV range. Surf. Interface Anal. 1994, 21, 165-176.

(17) Cappel, U. B.; Svanstro, S.; Philippe, B.; Giangrisostomi, E.; Ovsyannikov, R.; Leitner, T.; Fo, A.; Rensmo, H. Partially Reversible Photoinduced Chemical Changes in a Mixed-Ion Perovskite Material for Solar Cells. ACS Appl. Mater. Interfaces 2017, 9, 34970.

(18) Hutter, J.; Iannuzzi, M.; Schiffmann, F.; VandeVondele, J. cp2k: atomistic simulations of condensed matter systems. Wiley Interdiscip. Rev.: Comput. Mol. Sci. 2014, 4, 15-25.

(19) Nosé, S. A unified formulation of the constant temperature molecular dynamics methods. J. Chem. Phys. 1984, 81, 511-519.

(20) Perdew, J. P.; Burke, K.; Ernzerhof, M. Generalized Gradient Approximation Made Simple. Phys. Rev. Lett. 1996, 77, 3865-3868.

(21) Grimme, S.; Antony, J.; Ehrlich, S.; Krieg, H. A consistent and accurate $\mathrm{ab}$ initio parametrization of density functional dispersion correction (DFT-D) for the 94 elements H-Pu. J. Chem. Phys. 2010, 132, 154104.
(22) Grimme, S.; Ehrlich, S.; Goerigk, L. Effect of the damping function in dispersion corrected density functional theory. J. Comput. Chem. 2011, 32, 1456-1465.

(23) Lippert, G.; Hutter, J.; Parrinello, M. A hybrid Gaussian and plane wave density functional scheme. Mol. Phys. 1997, 92, 477-487.

(24) Goedecker, S.; Teter, M.; Hutter, J. Separable dual-space Gaussian pseudopotentials. Phys. Rev. B: Condens. Matter Mater. Phys. 1996, 54, 1703-1710.

(25) Krack, M. Pseudopotentials for $\mathrm{H}$ to $\mathrm{Kr}$ optimized for gradientcorrected exchange-correlation functionals. Theor. Chem. Acc. 2005, $114,145-152$.

(26) VandeVondele, J.; Hutter, J. Gaussian basis sets for accurate calculations on molecular systems in gas and condensed phases. $J$. Chem. Phys. 2007, 127, 114105.

(27) Poglitsch, A.; Weber, D. Dynamic disorder in methylammoniumtrihalogenoplumbates (II) observed by millimeter wave spectroscopy. J. Chem. Phys. 1987, 87, 6373-6378.

(28) Iannuzzi, M.; Hutter, J. Inner-shell spectroscopy by the Gaussian and augmented plane wave method. Phys. Chem. Chem. Phys. 2007, 9, 1599.

(29) Endres, J.; Egger, D. A.; Kulbak, M.; Kerner, R. A.; Zhao, L.; Silver, S. H.; Hodes, G.; Rand, B. P.; Cahen, D.; Kronik, L.; et al. Valence and Conduction Band Densities of States of Metal Halide Perovskites: A Combined Experimental-Theoretical Study. J. Phys. Chem. Lett. 2016, 7, 2722-2729.

(30) Schuster, O.; Wientjes, P.; Shrestha, S.; Levchuk, I.; Sytnyk, M.; Matt, G. J.; Osvet, A.; Batentschuk, M.; Heiss, W.; Brabec, C. J.; et al. Looking beyond the Surface: The Band Gap of Bulk Methylammonium Lead Iodide. Nano Lett. 2020, 20, 3090-3097.

(31) Wernet, P.; Nordlund, D.; Bergmann, U.; Cavalleri, M.; Odelius, M.; Ogasawara, H.; Naslund, L. A.; Hirsch, T. K.; Ojamae, L.; Glatzel, P.; et al. The Structure of the First Coordination Shell in Liquid Water. Science 2004, 304, 995.

(32) Vaz da Cruz, V.; Gelmukhanov, F.; Eckert, S.; Iannuzzi, M.; Ertan, E.; Pietzsch, A.; Couto, R. C.; Niskanen, J.; Fondell, M.; Dantz, M.; et al. Probing hydrogen bond strength in liquid water by resonant inelastic X-ray scattering. Nat. Commun. 2019, 10, 1013. 